\title{
USING MULTI RESOLUTION CENSUS AND RANKLET TRANSFORMATION IN LONG BASE LINE SAR IMAGE MATCHING
}

\author{
M. A. Ghannadi ${ }^{\text {a, }}$, M. Saadat Seresht ${ }^{\text {a }}$, M. Motagh ${ }^{\text {b }}$, A. Eftekhari ${ }^{\text {a }}$ \\ ${ }^{a}$ Dept. of surveying and Geomatics engineering, University of Tehran, Tehran, Iran - (amin.ghannadi84, \\ msaadat@gmail.com, akrameftekhary ) \\ ${ }^{\mathrm{b}}$ Helmholtz Centre Potsdam, GFZ German Research Centre for Geosciences, Potsdam, Germany - \\ Motagh@gfz-potsdam.de
}

KEY WORDS: SAR image matching, Census transformation, Ranklet transformation, FBM

\begin{abstract}
:
Stereo radargrammetry is a mature technique for deriving height information from SAR image pairs. Generally height derived method by Radargrammetry consists of two stages: Images matching and space intersection. In this paper we propose a multi-step image matching algorithm founded on feature based matching. In this multi step algorithm, a SAR image is firstly filtered by a speckle suppression algorithm. a SIFT (Scale invariant feature transform) is used to extract feature points. Then we use non parametric Transformation as discriptor for the points extracted. Matching is sometimes more efficient when operating on image signals that have been transformed in some way, rather than operating on the pure intensity values themselves; In this article we use a pair of spotlight long base line TerraSAR-X images from JAM (IRAN). In a part with $700 \times 700$ pixels of these images 90 points are matched with using Ranklet algorithm. The mean absolute error of the corresponding points is 0.9 pixel. This match points number is 49 points with using multi resolution Census. The result shows that our proposed multi step image matching is superior to the Most FBM methods in terms of accuracy and number of matched points.
\end{abstract}

\section{INTRODUCTION}

DSMs have large relevance in many engineering, land planning and environmental applications for a long time. An important part of the international research and development programs are related to the DSMs generation from satellite data, both optical and SAR. DSMs extraction from satellite stereo pair offers some advantages, among which low cost, speed of data acquisition and processing, availability of several commercial software and algorithms for data processing. "In particular, the DSMs generation from SAR data offers the significant advantage of possible acquisition during the night and in presence of clouds" (P. Capaldo et al, 2010).

Up to now, SAR imagery have been employed for the DSM generation using mainly the interferometric approach; to this aim, even special mission have been set up, as the Shuttle Radar Topography Mission (SRTM) or TanDEM-X mission, specially designed to yield elevation data on world scale. In the last years, thanks to the very high resolution imagery acquired by new sensors, as COSMO-SkyMed or TerraSAR-X, able to supply imagery with $1 \mathrm{~m}$ ground resolution, the radargrammetric approach became a valid alternative to generate DSMs from SAR data. In particular this method could be a resource to fill the gaps due to the lack of coherence in interferometric DSMs, in order to obtain the best (accurate and complete) product (Crosetto and Aragues, 1999).

The availability of new high resolution radar spaceborne sensors offers new interesting potentialities for the geomatics application: spatial and temporal change detection, features extraction, generation of Digital Surface Model. Stereo radargrammetry is a mature technique for deriving height information from SAR image pairs. Because stereo radargrammetry is less sensible to temporal decorrelation, it can provide better results than interferometric SAR in certain situations. Generally height calculating method by
Radargrammetry consists of two stages: Images matching and space intersection. In this paper we propose a multi-step image matching algorithm founded on feature based matching.

\section{MULTI-STEP IMAGE MATCHING ALGORITHM}

In this multi step algorithm, a SAR image is firstly filtered by a speckle suppression algorithm. a SIFT (Scale invariant feature transform) is used to extract feature points. Then we use non parametric Transformation as discriptor for the points extracted; at the end we use local sterategy for image matching.

\subsection{Speckle Suppression Algorithm}

Speckle is modeled as a multiplicative noise, which degrades the image and complicates clinical diagnosis. One of the best and earliest attempts to suppress speckle noisewasmade by averaging of uncorrelated images obtained from different viewpoints. These techniques involve hardware modifications that make the imaging process expensive and inconvenient. Another approach to speckle reduction is the use of postprocessing techniques, such as median, Lee, Frost, Kuan, and Gama filters. It has been demonstrated that these filters have major limitations. In this article we use Wiener filter for Speckle Suppression(Mohamad Forouzanfar et al, 2009)

\subsection{Feature Extraction}

Generally feature extraction methods used in optical images can take in SAR images. Because of speckle noise, some of these methods are not suitable for SAR images. Therefore, if the speckle noise is reduced, some of feature extraction methods can be used. Among these algorithms Harris and Forstner are performed to point features and gradient based algorithm are used on the SAR images edge detection(Pan, Chen et al. 2010). 
Scale Invariant Feature Transform (SIFT) is one of the most popular algorithm that is applied to SAR images for feature extraction. A strategy based on SIFT matching algorithm that initially feature based process for optical pattern recognition in images is presented. SIFT algorithm is independent from rotation, illumination changes, $3 \mathrm{D}$ viewpoint changes and affine distortion. Also it is stable against noise and illumination. Therefore it applied to feature extraction of SAR images. This algorithm consists of four major stages: Scale-space extreme detection, key point localization, Orientation assignment and key point descriptor (Lowe, 2004).

\subsection{Point Features Discriptor}

Matching is sometimes more efficient when operating on image signals that have been transformed in some way, rather than operating on the pure intensity values themselves; Examples here are the nonparametric Census and Rank. In some cases we can treat data as simple series of bits, each bit conveying some specific information. For instance an intensity signal can be preprocessed by a nonparametric Census transformation. In this case each bit reads whether a given intensity value is less, or is not, than a reference value. In such cases it is better to compare bit strings with binary measures, for example Hamming distance. Let us assume that a region around a central pixel was selected in an image. The Rank transform is defined as the number of pixels in that region for which the intensity signal is greater than or equal to the central one. The Census transform is an ordered stream of bits where each bit conveys information on whether the intensity of a central pixel is greater than a pixel from its neighbourhood or not. One of the Rank transformat is Ranklet transformation. The ranklet transform of an image I involves orientation-selective and non-parametric analysis. Orientation-selective analysis is then achieved by splitting half of the $\mathrm{N}$ pixels of each square crop into a subset $\mathrm{T}$ and the remaining half into a subset $\mathrm{C}$, these pairs of subsets being defined differently depending on the orientation considered.
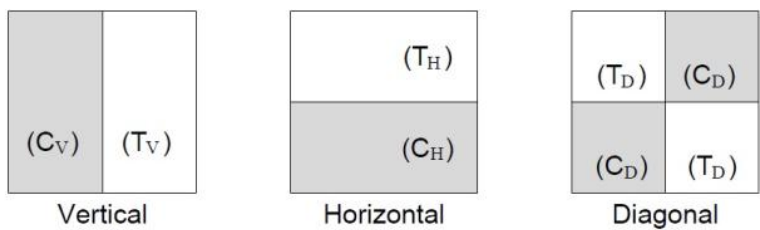

Figure 1. Ranklet transform, orientation-selective analysis From left to right, the vertical, horizontal, and diagonal pairs of subsets.

Finally, for each resolution and orientation, non-parametric analysis is carried out on each square crop. By means of $\mathrm{N} / 2 \times \mathrm{N} / 2=\mathrm{N} 2 / 4$ direct comparisons, the number of pixels pairs (pm, pn) satisfying the condition that gray-scale value of pm $2 \mathrm{~T}$ is higher than that of pn $2 \mathrm{C}$ can be calculated; This number would naturally range from 0 to $\mathrm{N} 2 / 4$ but, by appropriate normalization, can be mapped into the range(Matteo Masotti, Renato Campanini, 2008).

We match Corresponding points by multi resolution Census transformation and ranklet transformation as discriptor. Multiresolution analysis is achieved by choosing a set of resolutions, then extracting from the image I a variable size of central pixel neighbor window(B.Cyganek, J.P.Siebert, 2009).

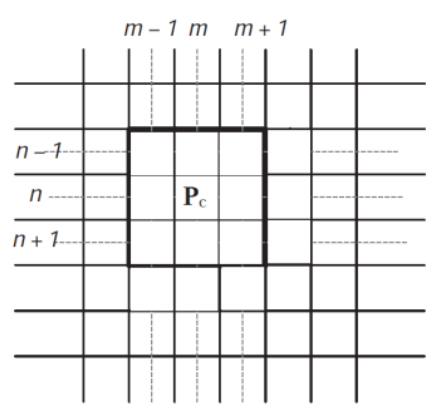

Figure 2. generate Census discriptor with comparing centeral pixel $(\mathrm{Pc})$ signal intensity and neighbors

\subsection{Proposed Method}

In this multi step algorithm, a SAR image is firstly filtered by a speckle suppression algorithm. a SIFT (Scale invariant feature transform) is used to extract feature points. Then we use non parametric Transformation as discriptor for the points extracted. Finally, for the evaluation this method we use the epipolar geometry and compared the method with one of the classical methods.

The multi step algorithm general procedure is shown below.

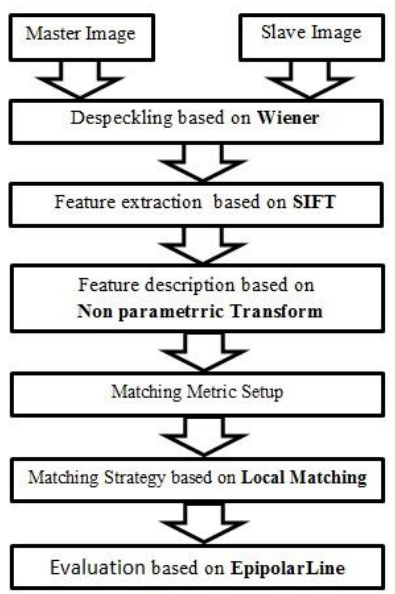

Figure 3. The multi step algorithm

\section{DATASETS AND RESULTS}

\subsection{Datasets}

In this study, we use a pair of TerraSAR-X Single-Look SlantRange Complex (SSC) images with long baseline. The images were acquired over the city of Jam, southern Iran, in spotlight mode and descending orbit. We use a part of these images by $700 \times 700$ pixels. These images are shown in Figure2. The ground elevation in the study area is between 620 and $680 \mathrm{~m}$. In the experiment, a total of 4 distinct ground control points in the four corners of images were measured by GPS. The accuracy of these points is less than $10 \mathrm{~cm}$. These points are used to refine RPCs. The image coordinates of these 4 points were carefully measured up to a nominal accuracy of 1 pixel. Also DEM by 1 meters resolution and 0.5 meter accuracy are used to evaluate the results in this research. 


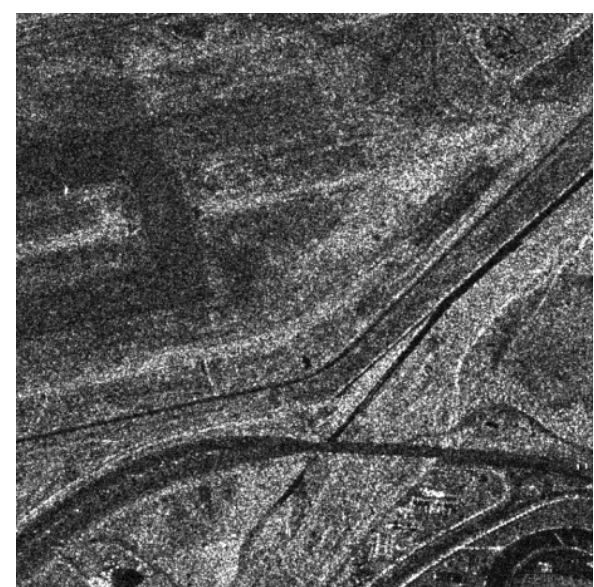

A

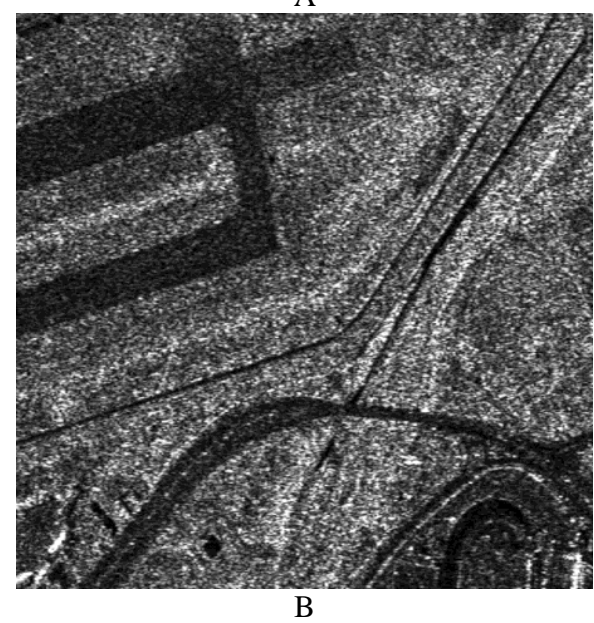

Figure 4. Master (A) and Slave (B) images by size $700 \times 700$ pixels that used in this study

\subsection{Results}

Test results are shown in Figure5 and Table 6. Ranklet transform and multi resolution Census are compared in Table 6.
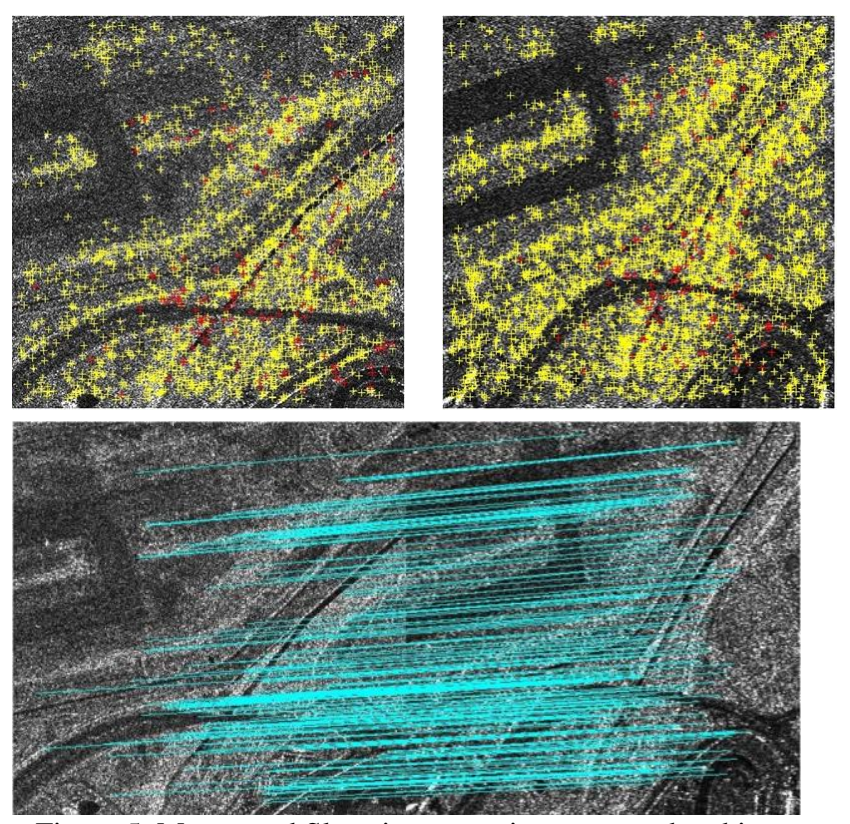

Figure 5. Master and Slave images points extracted and image matched with proposed multi step method using Ranklet

\begin{tabular}{|c|c|c|}
\hline Discriptor & $\begin{array}{c}\text { Num of Match } \\
\text { Points }\end{array}$ & $\begin{array}{c}\text { Matching } \\
\text { Accuracy }\end{array}$ \\
\hline Ranklet & 90 & 0.9 pixel \\
\hline ML Census & 49 & 1 pixel \\
\hline
\end{tabular}

Table 1. Statistical result of methods

\section{CONCLUTION}

The availability of new high resolution radar spaceborne sensors offers new interesting potentialities for the geomatics application: spatial and temporal change detection, features extraction, generation of Digital Surface Model. Stereo radargrammetry is a mature technique for deriving height information from SAR image pairs. Because stereo radargrammetry is less sensible to temporal decorrelation, it can provide better results than interferometric SAR in certain situations. Generally height calculating method by Radargrammetry consists of two stages: Images matching and space intersection. In this paper we propose a multi-step image matching algorithm founded on feature based matching. In this multi step algorithm, a SAR image is firstly filtered by a speckle suppression algorithm. a SIFT (Scale invariant feature transform) is used to extract feature points. Then we use non parametric Transformation as discriptor for the points extracted, we use local matching as matching sterategy. Finally, for the evaluation this method we use the epipolar geometry. The result shows that our proposed multi step image matching is superior to the Most FBM methods in terms of accuracy and number of matched points. Using Ranklet transform in multi step image has better results than using multi resolution Census in algorithm.

\section{REFRENCES}

B.Cyganek, J.P.Siebert, (2009), "An Introduction to 3D Computer Vision Techniques and Algorithms", pp.209-216.

Crosetto, M. and Aragues, F. P., (1999), "Radargrammetry and SAR interferometry for DEM generation: validation and data fusion", Proceedings of CEOS SAR Workshop, ESA-CNES, Toulouse, France.

F.Fayard, S.Meric, E.Pottier, (2010), "Generation of DEM by radargrammetric techniques," in Geoscience and Remote Sensing Symposium (IGARSS), IEEE International, pp. 43424345 .

Lowe, D. G. (2004). "Distinctive image features from scaleinvariant keypoints." International journal of computer vision 60(2): 91-110.

Matteo Masotti, Renato Campanini, (2008), " Texture classification using invariant ranklet features ", Department of Physics, University of Bologna Viale Berti-Pichat 6/2, 40127, Bologna, Italy, Article published in Pattern Recognition Letters 29, 1980-1986.

Mohamad Forouzanfar, Hamid Abrishami Moghaddam, Masoumeh Gity, (2009), " A new multiscale Bayesian algorithm for speckle reduction in medical ultrasound images ", SpringerVerlag London Limited.

P.Capaldo, M.Crepsi, F.Fratacangli, A.Nascetti, F.Pieralice, (2010), "DSM Generation From High Resolution Cosmo- 
International Archives of the Photogrammetry, Remote Sensing and Spatial Information Sciences, Volume XL-1/W3, 2013 SMPR 2013, 5 - 8 October 2013, Tehran, Iran

Skymed Imagery With Radargrammetri Model", Area Geodesy Geomatica-DICEA, University Rome, Italy. 